\title{
BASIC PSYCHOLOGICAL FACTORS AFFECTING ATHLETES' TRAINING
}

\author{
NADIIA VYSOCHINA1, ANASTASIIA VOROBIOVA² \\ ${ }^{I}$ National Defence University of Ukraine named after Ivan Chernyakhovskyi, Educational and Scientific \\ Institute of Physical Culture and Sports and Health Technologies, Kyiv, Ukraine \\ ${ }^{2}$ National University of Ukraine on Physical Education and Sport, Health, Fitness and Recreation Department, \\ Kyiv, Ukraine
}

\author{
Mailing address: Vorobiova Anastasiia, National University of Ukraine on Physical Education and Sport, Health, \\ Fitness and Recreation Department, 1 Fizkultury Street, 03150 Kyiv, Ukraine; tel. +38 044 2873261, \\ e-mail: anastasiia-99@bigmir.net
}

\begin{abstract}
Introduction. The paper aims to identify and systematise basic psychological factors which can influence athletes' training and competitive activity. Material and methods. In order to achieve the aim of the study, we carried out an expert survey and analysed its results statistically. The survey, whose concordance coefficient was 0.78 , was completed by 12 experts in the field (sports scientists and highly qualified athletes). Results. The study of the scientific and methodological literature about psychological correction taking into account the practice of athletes' training made it possible to formulate and systematise a number of key psychological factors (volitional, strategic, perceptive-aimed, anticipatory, managerial, psychophysiological, intellectual, introspective, and motivational) that affect the mental state and training process of athletes. The content and forms of the manifestation of psychological factors during competitive activity are examined, and their interrelations are studied. Integral indicators of psychological factors which influence competitive activity in Olympic sports are determined, and their priority of importance is examined. Conclusions. According to the experts' opinions, among the integral indicators of the psychological factors influencing athletes' activity, the leading ones are motivational, volitional, and psychophysiological factors. The results of the expert survey concerning the psychological factors influencing Olympic sports (game, cyclic, and complex coordination sports as well as martial arts) are analysed.
\end{abstract}

Key words: psychological factors, mental state, athletic training, competitive activity, Olympic sports

\section{Introduction}

The intensive development of psychotechnologies in modern conditions and their use as an instrument for increasing the effectiveness of athletic performance have caused more attention to be paid to seeking and developing new methods of psychological preparation and psychocorrection in sports. Nowadays, a new scientific foundation for psychotherapeutic intervention is being established in sport psychology. It is a synthesis of psychotherapeutic techniques, various directions of training, and psychological counselling in combination with national, cultural, and scientific traditions. This makes it necessary to systematise the knowledge accumulated in the field of psychological correction of the athlete's personality allowing them to make full use of their psychological abilities and increase the effectiveness of their sports performance.

Athletes' psychological capabilities are a common subject of research in modern Olympic sports. Attention is increasingly being paid to finding additional sources of emotional-volitional resources and athletes' internal mental reserves [1], because the level of their physical loads, record scores, and functional capabilities today have almost reached their limit. Further improvement of results can only be possible only with rational, scientifically grounded selection of adequate methods of psychological influence which must be based on the integration of individual methods into a holistic system of psychological correction [2].
This approach is one of the priorities of modern sports psychology.

The effectiveness of various methods of psychocorrection in elite sports $[3,4,5,6]$, experience in the complex use of psychotherapeutic methods $[7,8]$, as well as the optimisation of the training process by means of modern psychotechnologies [5, 9] are all addressed in the literature. However, the development of psychological correction programmes taking into account the current trends and the specificity of training and competitive processes is needed $[10,11]$.

The lack of a systematic approach to the application of psychological correction in athletic training is an unsolved but important issue related to psychological support for athletes practising Olympic sports.

\section{Aim and objectives}

The aim of the research is to identify and systematise basic psychological factors which can influence athletes' training and competitive activity.

The following objectives have been defined according to the above aim: to explore psychological factors affecting athletic training in Olympic sports and to substantiate their role in elite athletes, to assess the significance of psychological factors in the athletes' activities, as well as to analyse the correlation relationship between psychological factors. 


\section{Material and methods}

We analysed and generalised data from the relevant scientific and methodological literature and an expert survey, as well as applying methods of statistical analysis.

The expert survey included such stages as organising an assessment procedure, selecting experts, conducting surveys, and processing test results. The expert survey was carried out on the basis of the experts' ranking of the proposed psychological characteristics (volitional, motivational, psychophysiological, anticipatory, intellectual, strategic, perceptive-aimed, managerial, and introspective factors). The survey was completed by 12 experts, who were selected among participants of a forum for sports psychologists from Europe, taking into account their qualifications and experience. In order to receive more relevant results, the experts included not only researchers but also highly qualified athletes. Among 20 candidates who agreed to participate in survey, we selected twelve experts (the best specialists from Ukraine, Moldova, and Belarus) with a high level of skills in their field: six of them were specialists in the field of sports science (doctors of science, with extensive research experience), and six were highly qualified athletes (elite athletes, winners of world and European championships and other major competitions). The concordance coefficient was calculated to determine the consistency of the experts' opinions. According to this coefficient, the level of consistency between the experts' opinions $(\mathrm{W}=0.78, \mathrm{r}<0.01)$ was high. The survey was conducted during the 15th International Methodological Seminar on Sports Psychology (Minsk, 2018) using a questionnaire with the proposed factors which could have an influence on the psychological aspects of athletic training and competitive activity. The experts evaluated the importance of each factor for 4 groups of sports (game, cyclic, and complex coordination sports as well as martial arts) using a 10-point scale, where 0 indicated the lowest level, and 10 indicated the highest one. We analysed the influence of each factor on different groups of sports as well as the influence of those factors on athletes in sports in general (by examining the answers of experts for all 4 groups of sports).

The statistical measures used included the concordance coefficient (W), Pearson's correlation coefficient (r), and arithmetic means.

\section{Results}

In modern sport psychology, there are many areas of psychological correction, which are characterised by a great variety of approaches. Consequently, it is necessary to select psychological means and methods which will be adequate to the aim of psychocorrection and correspond to the conditions of competitive activity. In addition, one must take into account their complementarity and integral nature of their impact, which will make it possible to create individual psychological training programmes for increasing the effectiveness of athletes' competitive activity at different stages of long-term improvement in various kinds of sports [12].

The key psychocorrection approaches according to our point of view are psychocorrection of emotional states in sports activities, correction of the level of psychological self-regulation of the athlete's personality, correction of mental cognitive processes of athletes, psychocorrection of interpersonal relationships of athletes, and correction of the goal-setting system of athletes through coaching [13].

The study of the scientific and methodological literature about psychological correction in the training process allowed us to formulate and systematise a number of key psychological factors that affect the athlete's mental state; these are volitional, motivational, psychophysiological, anticipatory, intellectual, strategic, perceptive-aimed, managerial, and introspective factors. Most of the proposed psychological factors are unexplored, which motivated us to give them more attention and describe our conceptualisations of these factors.

In order to understand the relationships between psychological factors which influence athletes' activity, we first analysed the correlations between (Tab. 1). The results of this analysis indicated that the motivational factor correlated the most with the other factors, especially those having a behavioural component; these were the following: the strategic $(\mathrm{r}=1.00)$, perceptive-aimed $(\mathrm{r}=0.67)$, anticipatory $(\mathrm{r}=0.99)$, and managerial $(r=0.71)$ factors. The close correlation between the motivational and strategic factors $(\mathrm{r}=1.00)$ could be explained by the connection between the sequence of an athlete's thoughts and actions with regard to obtaining a certain result with the reasons that motivate the athlete to act. The stronger the moti-

Table 1. Correlations between psychological factors influencing athletes' activity

\begin{tabular}{|c|c|c|c|c|c|c|c|c|c|c|}
\hline No & Psychological factors & I & II & III & IV & V & VI & VII & VIII & IX \\
\hline 1. & Volitional & 1.00 & & & & & & & & \\
\hline 2. & Strategic & -0.19 & 1.00 & & & & & & & \\
\hline 3. & Perceptive-aimed & 0.29 & $0.63^{*}$ & 1.00 & & & & & & \\
\hline 4. & Anticipatory & 0.23 & $0.99^{*}$ & $0.75^{*}$ & 1.00 & & & & & \\
\hline 5. & Managerial & $0.87^{*}$ & $0.64^{*}$ & $0.59^{*}$ & $0.67^{*}$ & 1.00 & & & & \\
\hline 6. & Psychophysiological & $0.63^{*}$ & 0.06 & 0.43 & -0.03 & 0.47 & 1.00 & & & \\
\hline 7. & Intellectual & 0.49 & 0.32 & $0.91^{*}$ & 0.47 & $0.60^{*}$ & -0.35 & 1.00 & & \\
\hline 8. & Introspective & $0.81^{*}$ & 0.25 & 0.33 & 0.28 & -0.48 & $-0.86^{*}$ & 0.06 & 1.00 & \\
\hline 9. & Motivational & 0.28 & $1.00^{*}$ & $0.67^{*}$ & $0.99 *$ & $0.71^{*}$ & 0.09 & 0.39 & 0.19 & 1.00 \\
\hline
\end{tabular}

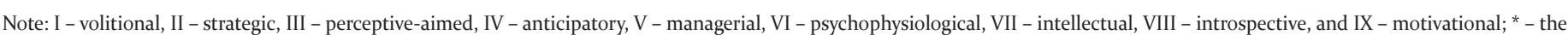
correlation coefficient is significant at the level of $\mathrm{p}<0.05$. 
vation and the better the understanding of the tasks, the more accurately the strategy for achieving the goal is formed. It should be noted that all these factors have significant positive correlations among themselves. In addition, the perceptive-aimed and managerial factors have positive relationships with the intellectual factor ( $r=0.91$ and $r=0.60$, respectively), which indicates the need for an analytical approach in these areas.

At the same time, the volitional factor has a high level of correlation with the introspective one $(r=0.81)$, which indicates its reflexive character or the need of an internal comprehension of volitional attitudes. The psychophysiological factor has an inverse statistically significant correlation relationship with the introspective one $(\mathrm{r}=-0.86)$, which reflects the low indices of introspection in game and cyclic sports combined with high psychophysiological indices.
The results regarding the impact of psychological factors on the competitive activity of representatives of different sports, which was estimated by experts using a 10-point scale, are shown in Figure 1. The following types of factors had the highest level of influence on competitive activity for particular sports: motivational, anticipatory, and intellectual factors for game sports; volitional, motivational, and psychophysiological factors for cyclic sports; volitional, motivational, anticipatory, and introspective factors for martial arts; and motivational, intellectual, and introspective factors for complex-coordination sports.

The biggest difference between the four types of sports could be observed in cyclic sports. It can be explained by the clear structure of movements with practically no external factors that can affect the effectiveness of competitive activities,
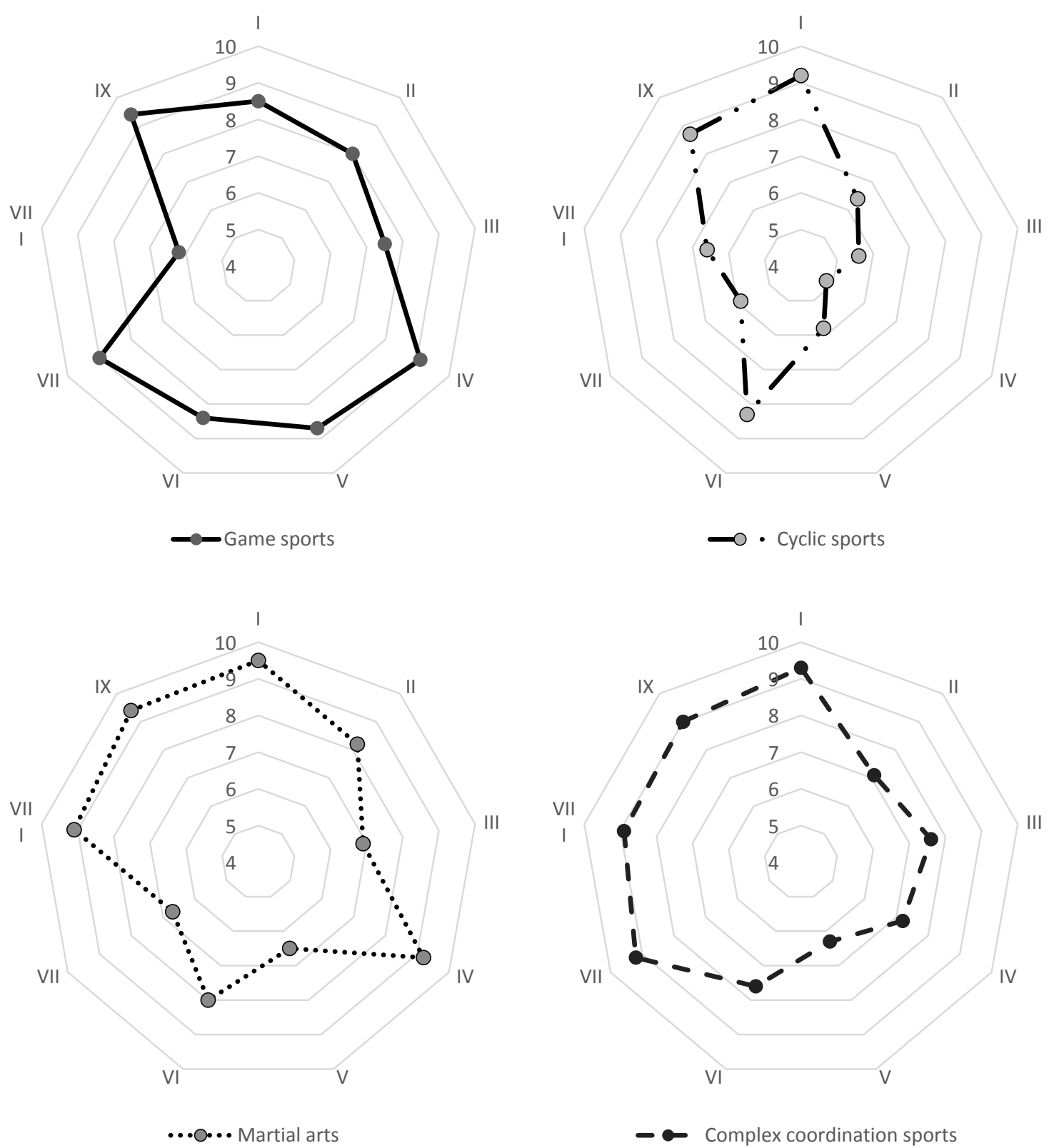

Figure 1. Influence of psychological factors on competitive activity of representatives of different sports according to expert survey results (on 10-point scale): I - volitional, II - strategic, III - perceptive-aimed, IV - anticipatory, V - managerial, VI - psychophysiological, VII - intellectual, VIII introspective, and IX - motivational. 
including the lack of the need to respond quickly to the actions of the opponent or to predict their movements.

The complex psychological factor impact was calculated as the amount of all factors for each group of sports (maximum 90 points). The greatest influence was found on competitive activity in game sports (74.8 points), followed by martial arts (73.5 points) and complex-coordination sports (72.2 points), and clearly the smallest value was obtained for cyclic sports (61.3 points).

\section{Discussion}

In the literature, the most explored psychological factors are volitional, motivational and psychophysiological in nature [4, 11, 14]. Less studied factors include anticipatory and intellectual factors $[6,15]$. On the other hand, strategic, perceptive-aimed, managerial, and introspective factors remain hardly explored.

To assess the significance of psychological factors in athletic activity, we studied the experts' opinions, and the priority of influence of the factors was determined on their basis (Fig. 2). Integral indicators of the main psychological factors were calculated based on the experts' ratings for each group of sports (game, cyclic, and complex coordination sports as well as martial arts) using a 10-point scale (the maximum score being 40 points).

In general, the analysis of the experts' opinions showed that the motivational factor (related to causes, or motives, and the extent to which they encourage the athlete to strive to win the competition) and the volitional factor (the psychological ability of an athlete to voluntarily mobilise volitional efforts to overcome obstacles, achieve goals, perform complex tasks, control emotions, etc.), which received 36.5 points each, were the leading integral indicators of the psychological factors which influenced athletes' activity $(\mathrm{p}<0.05)$. This confirms the established opinion [11, 14, 15].

The next important factor is the psychophysiological one (32.3 points), which characterises the genotypically conditioned properties of the athlete's psyche. In our study, the psychophysiological factor included the basic characteristics of the temperament type: strength and weakness, mobility and inertia, balance and imbalance of the nervous system according to I. Pavlov, as well as introversion-extraversion and neuroticism according to H. Eysenck [7]. In addition, the athlete's stress resistance (determined on the basis of indicators of psychological and physiological stress) and other indirect indicators are located in the zone of influence of this factor [11].

This factor was followed by the intellectual and introspective factors, which can be regarded as complementary and which had an equal influence on athletic activity (30.8 points each).

The intellectual factor is manifested in sports through the athlete's mental cognitive processes (thinking, attention, memory, etc.), which reflect their ability to cognise, understand and solve problems, and determine the effectiveness of a strategy of achieving a competitive goal. Thanks to it, the athlete can analyse game situations, the actions of the opponent, and their own mistakes; make conclusions; plan the preparation rationally; as well as abstract, quickly perceive, and process information, etc.

The introspective factor is the most in demand, in our opinion, in the modern athlete's psychological preparation [16]. Introspection involves observing one's own mental processes and experiences: thoughts, visualisations, and feelings. At the same time, self-analysis is carried out through reflection - immersion in one's own consciousness and rethinking of values, interests, motives, perceptions, reasons for decision-making, emotional reactions, behavioural patterns, etc.

In the absence of a correlation relationship between them, it is obvious that the degree of manifestation of self-knowledge or introspection is largely determined by the functions of thinking, attention, and memory. Vice versa, the more an athlete shows mindfulness and does psychological work, the higher their intellectual capabilities are. The lack of correlation between them in the current study is due to the fact that the intellectual factor predominates in game sports, and the introspective one dominates in martial arts.

According to the integral evaluation of the influence of psychological factors on the athlete's personality, less significant factors included anticipatory, strategic, perceptive-aimed, and managerial ones.

The anticipatory factor allows the athlete to intuitively anticipate the actions of the opponent, to demonstrate accurate

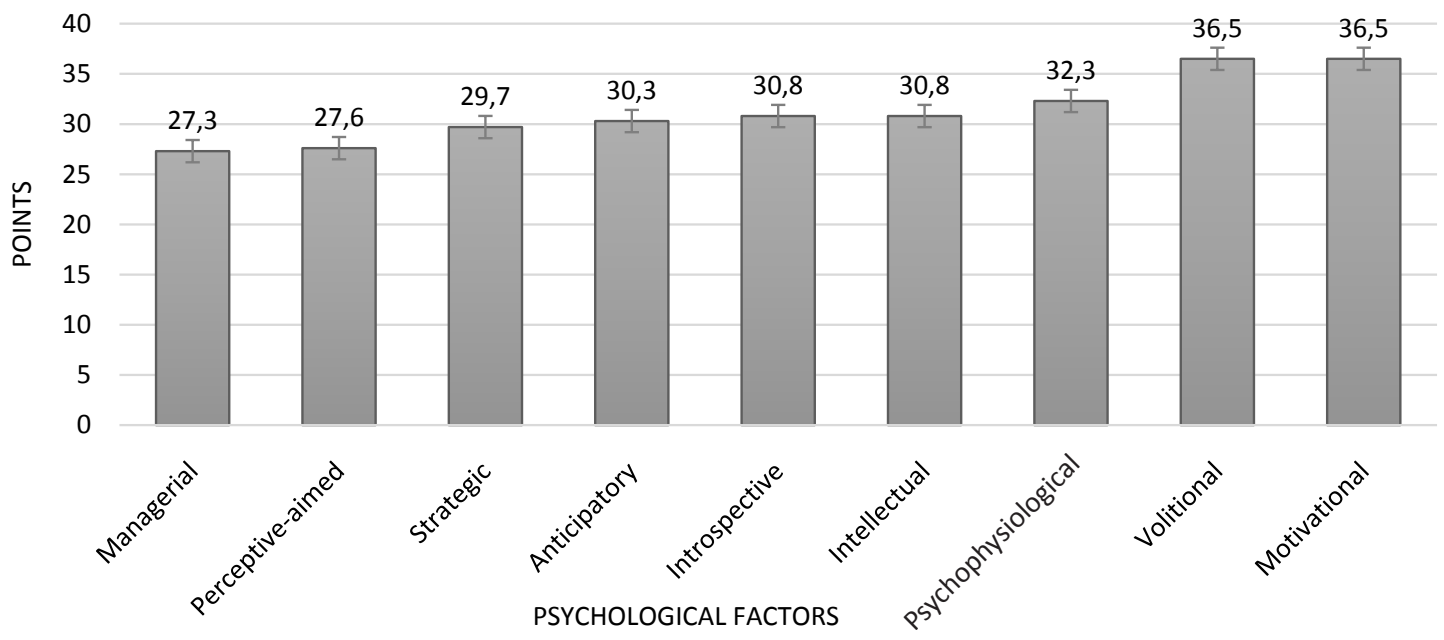

Figure 2. Integral indicators of main psychological factors affecting athletes' competitive activity 
forestall reactions (especially in martial arts and game sports), and helps to reduce the risk of injury. Athletes with a sensitive nervous system have well developed anticipation skills.

The strategic factor determines the sequence of thoughts and actions of the athlete aimed at obtaining a particular result. In our study, it influences the direction of the athlete's actions to create or destroy, the practical realisation of plans (practice) or ideological thinking (theory), as well as the predominance of strategic or tactical thinking. All these psychological aspects are important for the successful implementation of competitive activities, but realising the psychological potential by the athlete influences the choice of the way to achieve the desired result. For instance, performing the same technical element (such as scoring a goal in a game sport or striking in martial arts), the "destroyer" will have a mental attitude of destroying the opponent, demonstrating strength and aggression, and the "creator's" thoughts will be focused on the beauty and accuracy of movement, pleasure derived from the process of struggle, and professional success. Management of the strategic factor will be focused not on changing the athlete's attitude, but on maximising the adaptation of the athlete's present psychological characteristics to the conditions of their competitive activity.

The perceptive-aimed factor reflects the athlete's perception of their future (optimism-pessimism). An optimistic perception of life inclines the athlete to focus on the best aspects of people, things, and phenomena; to believe in themselves, in their victory, and the favourable outcome of events; to seek improvement; and not to lose heart in difficult situations. At the same time, excessive optimism does not allow the athlete to see obstacles and makes them neglect the significance of these obstacles, which can have both a positive and negative impact on the outcome of sport performance. A pessimistic perception is usually manifested in imaginary obstacles created by the athlete or in exaggerating the importance of real problems, which typically leads to anxiety and increased stress, especially when experiencing high physical and psychoemotional loads. On the other hand, pessimism helps to see the difficulties in achieving the goal, and thanks to this, programmes can be developed to overcome these difficulties.

The managerial factor characterises the role of the athlete in the team and their social functions (teacher-student, leader-follower, or ideologist-performer). This factor is present in almost all sports, but it is mostly expressed in team game sports, as it is directly related to the role the athlete plays in the team, being, for example, a forward or a defender in football, ice hockey, and basketball or the skip (team captain) in curling, who makes strategic decisions. This factor more often has an indirect character, expressed in the interaction with the coach and colleagues, and depends on the volitional, motivational, and psychophysiological characteristics of the athlete.

However, the place and contribution of these factors in the overall structure of psychological factors indicate that they must be taken into account in developing programmes for the psychological preparation of athletes. It is interesting to note the behavioural component of these factors. Anticipation is manifested in the intuitive reactions of an athlete, especially under limited time conditions. Optimism or pessimism, which characterise the perceptive-aimed factor, shape a characteristic way of thinking and determine the choice of actions (for example, in an unfavourable competitive situation, the athlete may surrender and stop making efforts or fight). The strategic and managerial factors reflect the athlete's behavioural style, which shows the way they achieve success. Here lie the reserves and prospects for scientific research in the psychology of sports in our opinion.

\section{Conclusions}

This study has sought to determine the main psychological factors which influence athletic training. The content and forms of their manifestation in competitive activity are examined. Based on the expert survey, the most significant factors were motivational, volitional, and psychophysiological ones. The factor argued to be the most in demand and worth further study is the introspective factor, because it determines the degree of athletes' awareness, meaningful attitude to work, and vision of the goal, and it is undeservedly underestimated and often ignored in practice. Thanks to the ability of introspection, the athlete can analyse their errors qualitatively, which can help them reduce their energy costs in future performances, prevent new errors, and learn to recognise their strengths and weaknesses, giving them an undoubted advantage over the opponent.

The integral indicators of psychological factors which impact competitive activity in Olympic sports were calculated and analysed. Their interrelations and priority of importance were examined. The local influence of psychological factors on the performance of athletes in different types of Olympic sports (game, cyclic, and complex-coordination sports and martial arts) were analysed as well.

Prospects for further research are related to the development of a system of athletes' personality psychocorrection in the process of sports training using knowledge about psychological factors.

\section{Literature}

1. Yakovlev B.P., Babushkin G.D., Apokin V.V. (2015). Psychological factors to contribute to effective implementation of athlete's capacities under competitive conditions. Theory and Practice of Physical Culture 11, 83-85.

2. Anshel M.H. (2002). Sport psychology: From theory to practice. San Francisco: Benjamin-Cummings Publishing Company, $450 \mathrm{p}$.

3. Johnson D. (2008). How psychology helps the athlete: Helping athletes with a wide range of issues, both on and off the field, to deal with the stresses of their game. Tribune Business News, McClatchy, 18 May 2008.

4. Kokun O.M. (2015). Professional self-fulfilment of skilled people of different professional groups and specialities. Social Welfare. Interdisciplinary Approach 5(2), 19-32.

5. Lemyre P.N., Treasure D.C., Roberts G.C. (2006). Influence of variability in motivation and affect on elite athlete burnout susceptibility. Journal of Sport and Exercise Psychology $28,32-48$.

6. Serova L.K. (2007). Psychology of the athlete's personality. Moscow: Sovetskiy sport, 116 [in Russian].

7. Eysenck H.J. (2013). Experiments in Personality: Volume 1. Psychodiagnostics and Psychodynamics. Abingdon: Routledge, 278.

8. Unestal L.E., Bundzen P., Malinin A. (1992). Integrated psychophysical training: Methodology and construction. Mental training for sport and life in Russia. Orebro, Sweden. 1416.

9. Vysochina N.L. (2013). Optimisation of the goal-setting system for athletes by coaching. Bulletin of Chernihiv National Pedagogical University 112(1), 75-78 [in Russian]. 
10. Platonov V.N. (2015). The system of training athletes in Olympic sports. General theory and its practical applications. Kyiv: Olympic literature, 752 [in Russian].

11. Williams J., Krane V. (2014). Applied sport psychology: Personal growth to peak performance (7th ed.). New York: McGraw-Hill.

12. Vysochina N. (2016). Psychological support in long-term preparation of athletes. Sporto mokslas 4(86), 2-9. DOI: $10.15823 / \mathrm{sm} .2016 .36$.

13. Vysochina N., Vorobiova A. (2017). The system of psychological correction of athlete's personality in Olympic sport. Sporto mokslas 4(90), 35-45. DOI: http://dx.doi. org/10.15823/sm.2017.37.

14. Beckmann J., Fröhlich S.M., Elbe A.M. (2009). Motivation und volition. In W. Schlicht, B. Straus (eds.), Grundlagen der sport psychologie (pp. 511-562). Göttingen: Hogrefe.

15. Horn T.S. (2008). Advances in sport psychology ( $3^{\text {rd }}$ ed.). Champaign, IL: Human Kinetics.

16. Yakovlev B.P., Babushkin G.D., Babushkin E.G. (2015). Psychological support of pre-season training of preparation of highly skilled athletes. Theory and Practice of Physical Culture (7), 27-27.

Submitted: February 21, 2019.

Accepted: June 17, 2019. 